\title{
Effect of soluble dietary fibre on the viscosity of gastrointestinal contents and the acute glycaemic response in the rat
}

\author{
BY D. CAMERON-SMITH, G. R. COLLIER* AND K. O'DEA \\ Department of Human Nutrition, Deakin University, Geelong, Victoria, 3217, Australia
}

(Received 4 January 1993 - Revised 11 June 1993 - Accepted 8 July 1993)

\begin{abstract}
The postprandial glycaemic response following a meal is reduced with the addition of soluble dietary fibre. The reductions in the glycaemia are thought to be due largely to increased viscosity of the gastrointestinal (GI) contents retarding digestion and absorption. The aims of the present study were to determine the effect that the GI tract has on the viscosity of meals containing different soluble fibres and to determine whether the glycaemic response of a meal (containing the soluble fibre) was predicted by the viscosity of the digesta in the small intestine. High carbohydrate diets containing $70 \mathrm{~g}$ soluble fibre (guar gum, xanthan gum or methylcellulose) $/ \mathrm{kg}$ or $70 \mathrm{~g}$ insoluble fibre (wheat bran) $/ \mathrm{kg}$ were diluted in water to a final fibre concentration of $18 \mathrm{~g} / \mathrm{kg}$. Following dilution the wheat bran diet had no measurable viscosity, while the viscosities of the soluble fibre diets were elevated. When the diets were fed to male Sprague-Dawley rats for 2 weeks the viscosities of the stomach and small intestinal digesta were not predicted by the viscosity of the diets measured before ingestion. The action of the GI tract on the viscosity of the soluble fibres was investigated in vitro by dilution of the diets with acidic and neutralizing solutions, mimicking gastric and duodenal secretions. Dilution of diets with either acidic and neutralizing solutions or saline control significantly lowered the viscosity of all diets, while alterations in the $\mathrm{pH}$ of the diets had little impact on the resultant viscosity. When fasted rats were orally administered with the differing diets $(0.25 \mathrm{~g}$ carbohydrate/ $\mathrm{kg}$ body weight), the postprandial glucose response was reduced following the soluble-fibre-containing meals when compared with the wheat bran-supplemented meal, although the reduction in glycaemia only reached statistical significance with xanthan supplementation. These results indicate that there are large changes in the viscosity of a meal containing soluble fibre following ingestion, and that dilution of the diet by GI secretions is important in determining the resultant viscosity in the small intestine. Furthermore, the large differences in viscosity of the GI contents following consumption of the diets containing the soluble fibres were not predictive of the postprandial glycaemic response.
\end{abstract}

Soluble fibre: Viscosity: Gastrointestinal contents: Rat

It is now widely recognized that the postprandial glycaemic response to a meal is reduced with the addition of soluble dietary fibre, in both normal and diabetic subjects (Jenkins et al. 1977; Goulder et al. 1978). The greatest reduction in the glycaemic response to a meal is observed with soluble fibres which hydrate rapidly and develop significant viscosity in solution (Jenkins et al. 1978; O'Connor et al. 1987). The addition of soluble fibre to a meal increases the viscosity of the meal and, following ingestion, increases the viscosity of the gastrointestinal (GI) contents (Blackburn \& Johnson, 1981; Lund et al. 1989). The increased viscosity of the meal retards the rate of carbohydrate digestion and absorption (Caspary et al. 1980; Blackburn \& Johnson, 1983; Flourie et al. 1984; Blackburn et al. 1984).

It has generally been accepted that the viscosity of guar gum and pectin preparations has been shown to predict the reduction in the glycaemic response (O'Connor et al. 1987;

* For reprints. 
Ebihara et al. 1981). However, using different fibre preparations Edwards et al. (1987) were unable to demonstrate a correlation between viscosity measured in vitro and the glycaemic response to the meal. Because the rate of small intestinal absorption is related to the viscosity of the digesta (Flourie et al. 1984) it is important to consider the actions of the secretions which enter the GI tract. Ingested food is diluted by saliva, acidic gastric juices, neutralizing exocrine pancreatic secretions and bile. Dilution of soluble fibres with acidic and neutralizing solutions mimicking GI secretions has been shown to have different effects on the fibres tested. The resultant viscosity following the dilutions was more closely correlated with the glycaemic response of the meal than the viscosity measured before dilution (Edwards et al. 1987). However, it is not known what effect the GI tract has on the viscosity of ingested meals containing soluble fibre and the correlation between the viscosity of the small intestinal contents and the glycaemic response to a meal.

The aim of the present study was to investigate the action of the GI tract on the viscosity of several viscous fibre types. The viscous fibres were fed in the diet to male Sprague-Dawley rats and the viscosity of the stomach and small intestinal contents measured. To determine whether the resultant viscosity of the digesta in the small intestine is due to $\mathrm{pH}$ changes and/or dilution by the secretions entering the GI tract, the diets were diluted in vitro with acidic and neutralizing solutions. The acute glycaemic response to a test meal was also measured in the rat and compared with the viscosities of the meal and the small intestinal digesta.

\section{MATERIALS AND METHODS}

\section{Viscosity measurements}

The viscosity of the soluble fibres in solution was measured in poise (P) at $37^{\circ}$ using a Brookfield Synchro-Lectric viscometer (model DV-II; Brookfield, Stroughton, USA). The viscosity was measured as the force required to overcome the resistance offered by the sample to the rotation of a cylinder (Spindle no. 4; Brookfield, USA) immersed in the solution (Sherman, 1970). Viscosities were determined in samples with a minimum volume of $80 \mathrm{ml}$, with the exception of the gastrointestinal samples which had minimum volumes of $25 \mathrm{ml}$. As the fibres investigated exhibited non-Newtonian behaviour in solution with differing degrees of shear thinning, unless otherwise stated the viscosity was measured at the viscometer's lowest shear rate $(0.005 \mathrm{rev} . / \mathrm{s})$ in order to minimize these differences.

\section{Diets}

The compositions of the non-viscous wheat bran control and experimental diets are given in Table 1 . As a percentage of total energy, the diets contained: carbohydrate $69 \%$, fat $19.5 \%$ and protein $11.5 \%$, while the fibre content of all diets was $70 \mathrm{~g} / \mathrm{kg}$. The coarse wheat bran (Bunge, Melbourne, Australia), guar-gum flour (Supercol G; Chemiplas, Epping, Australia), xanthan gum (Kelco, San Diego, USA) and methylcellulose (Sigma, St. Louis, USA) were all food grade quality.

\section{Viscosity of the stomach and small intestinal contents}

Twenty male Sprague-Dawley rats (200-240 g initial body weight) were housed in groups of five in a temperature-controlled room $\left(22 \pm 1^{\circ}\right)$ on a $12 \mathrm{~h}$ light-dark cycle. Each group of five rats was allocated to one of the experimental diets (Table 1) and fed ad lib. for 2 weeks. All rats were killed at 09.00 hours by cervical dislocation and the contents of the stomach and small intestine were collected. Stomach and small intestinal digesta were pooled to allow sufficient volume for accurate viscosity measurement. Due to the paste-like consistency of some samples a dilution of $1: 1(\mathrm{v} / \mathrm{v})$ with tap water was necessary for all the 
Table 1. Experimental diet composition $(\mathrm{g} / \mathrm{kg})$

\begin{tabular}{lcccc}
\hline \hline Ingredient & Wheat bran & Xanthan & Guar & Methylcellulose \\
\hline Maize starch & $637 \cdot 6$ & $693 \cdot 3$ & $693 \cdot 3$ & $693 \cdot 3$ \\
Casein & $159 \cdot 4$ & 180 & 180 & 180 \\
Butter & 38 & $45 \cdot 6$ & $45 \cdot 6$ & $45 \cdot 6$ \\
Mineral mix* & $4 \cdot 2$ & $4 \cdot 2$ & $4 \cdot 2$ & $4 \cdot 2$ \\
Vitamin mix $\dagger$ & $1 \cdot 2$ & $1 \cdot 2$ & $1 \cdot 2$ & $1 \cdot 2$ \\
Choline chloride & $0 \cdot 25$ & $0 \cdot 25$ & $0 \cdot 25$ & $0 \cdot 25$ \\
Methionine & $0 \cdot 35$ & $0 \cdot 35$ & $0 \cdot 35$ & $0 \cdot 35$ \\
Coarse wheat bran & 150 & - & - & - \\
Xanthan gum & - & 72 & - & - \\
Guar gum & - & - & 72 & 72 \\
Methylcellulose & - & - & - & - \\
\hline \hline
\end{tabular}

* Composition (g/kg of mix): $\mathrm{CaHPO}_{4} 623 \cdot 4, \mathrm{NaCl} 72 \cdot 8, \mathrm{~K}_{3} \mathrm{C}_{6} \mathrm{H}_{5} \mathrm{O}_{7} 216 \cdot 5, \mathrm{~K}_{2} \mathrm{SO}_{4} 51 \cdot 2, \mathrm{MgO}_{2} 3 \cdot 6, \mathrm{MnSO}_{4}$ $\left.5 \cdot 05, \mathrm{C}_{6} \mathrm{H}_{5} \mathrm{O}_{7} \mathrm{Fe} 5 \cdot 9, \mathrm{ZnO}_{4} 1 \cdot 02, \mathrm{CuSO}_{4} 0 \cdot 6, \mathrm{KIO}_{3} 0 \cdot 001, \mathrm{Na}_{2} \mathrm{SeO}_{3} 0 \cdot 005, \mathrm{Cr}\left[\mathrm{K}\left\{\mathrm{SO}_{4}\right\}_{2}\right]\right]_{0} \cdot 78$.

$\uparrow$ Colborn Dawes, Wagga Wagga, Australia: composition as recommended by the American Institute of Nutrition (1977).

samples. The viscosities of the GI contents were determined after the samples were heated to $37^{\circ}$.

\section{In vitro studies}

To determine whether changes in $\mathrm{pH}$ or dilution of food in the GI tract control the viscosity of digesta in the small intestine, we attempted in vitro to mimic the changes in $\mathrm{pH}$ and dilution that occur in the GI tract. Diets were prepared in tap water before being diluted first with an acidic solution and then with a neutralizing solution. The diets $(30 \mathrm{~g})$ were mixed thoroughly with $180 \mathrm{ml}$ tap water and incubated for $1 \mathrm{~h}$ before the first viscosity measurement. The measurement of viscosity $1 \mathrm{~h}$ after mixing was used to provide a measure of the viscosity changes that may occur in the GI tract following the consumption of freshly mixed or unhydrated fibre preparations. The diets were then diluted further by the addition of $22.5 \mathrm{ml} 0.1 \mathrm{M}-\mathrm{HCl}$ and $54 \mathrm{~mm}-\mathrm{NaCl}$ solution (acidifying the diluted diet solutions to $\mathrm{pH} 2$ ), incubated again for $\mathrm{l} \mathrm{h}$ and the viscosity measured again. The same diets were diluted further with $130 \mathrm{ml}$ neutralizing solution $\left(120 \mathrm{mM}-\mathrm{NaHCO}_{3}, 5 \mathrm{~mm}-\mathrm{KCl}\right.$ and $35 \mathrm{~mm}-$ $\mathrm{NaCl}$ ). The reneutralized diets in solution were incubated for a further $1 \mathrm{~h}$ after which the final viscosity measurement was made. To determine the effect of dilution in the absence of a $\mathrm{pH}$ change the diets were diluted with equivalent volumes of $150 \mathrm{~mm}-\mathrm{NaCl}$ and incubated under identical conditions.

\section{Acute glycaemic response}

Sixteen male Sprague-Dawley rats (approximately $200 \mathrm{~g}$ body weight) were divided randomly into groups of four and fed on a standard rat diet (Clark King and Co, Pakenham, Australia). The evening before measurement of the glucose response the diet was removed at 18.00 hours and the animals fasted overnight. At 09.00 hours the rats were given the experimental diets by oral administration $(0 \cdot 25 \mathrm{~g}$ carbohydrate $/ \mathrm{kg}$ body weight). The experimental diets, shown in Table 1, were modified so that glucose replaced half of the maize starch; the remaining ingredients of the diets were unaltered. The diets were diluted 1:4 (w/v) with tap water to give a consistency that enabled the diet to be administered orally. The animals were lightly anaesthetized with diethyl ether and the meal administered directly into the stomach. Tail vein blood samples $(50 \mu \mathrm{l})$ were taken from the 
conscious animal before the meal (time 0 ) and at 15,30,45, 60, 90, 120, and $180 \mathrm{~min}$ following the administration of the meal for blood glucose determination. Blood glucose was analysed immediately on a glucose analyser (Model 27; Yellow Springs Instrument Company, Yellowsprings, USA).

The formulation of the diets and all the experimental procedures used were formally approved by the Animal Ethics Committee, Deakin University.

\section{Statistical analysis}

The statistical significance of differences between means was established by two-way $t$ tests. Values of $P<0.05$ were accepted as statistically significant. Incremental areas under the curve were calculated geometrically using the trapezoid rule. Values below the fasting level were ignored.

\section{RESULTS}

\section{Viscosity of the stomach and small intestinal contents}

The stomach and small intestinal digesta extracted from the rats given the diet supplemented with wheat bran were of a watery consistency with viscosities too low to measure. Supplementation of the diet with soluble fibre yielded stomach and small intestinal digesta with considerable viscosity, shown in Fig. 1. The digesta extracted from the stomachs of all these animals were thick paste-like masses, which after dilution 1:1(v/v) with water had measurable viscosities. The small intestinal contents contained a greater fluid fraction; however, after dilution with water the viscosities were similar to those measured for the gastric digesta.

The effects of increasing rates of shear, by increasing the revolution rate of the spindle immersed in the solution, on the apparent viscosities of the small intestinal contents taken from the rats given the viscous fibres are shown in Fig. 2. With increasing rates of shear the viscosity of each of the fibre solutions was lowered. The reductions in viscosity with increased shear for guar gum and methylcellulose were similar. However, for xanthan the reductions in viscosity with increasing shear were substantial; increasing the shear from 0.005 to $0.01 \mathrm{rev} . / \mathrm{s}$ lowered the apparent viscosity by $59.8 \%$.

\section{In vitro studies}

The effects of diluting the diets with acidic and neutralizing solutions to mimic the actions of the GI tract are shown in Table 2. After dilution of the diets to a final fibre concentration of $18 \mathrm{~g} / \mathrm{kg}$, the most viscous diet contained xanthan which was approximately twice as viscous as guar (1210 (SE 34) v. 735 (SE 39) P respectively). These diets were very much more viscous than the methylcellulose diet $(6 \cdot 18$ (SE 0.6) P). The viscosity development of xanthan and methylcellulose was rapid, while for guar the viscosity increased as a function of time (results not shown). At $1 \mathrm{~h}$ after mixing the viscosity of the guar preparation was approximately $75 \%$ of the total viscosity after $24 \mathrm{~h}$ development.

The viscosity of the xanthan diet in tap water increased with the addition of either the acidic solution or saline $(P<0 \cdot 05)$. Dilution of the guar diet with the acidic solution resulted in a significantly increased viscosity $(P<0.05)$, while the viscosities of the equivalently diluted saline control samples were not increased and differed significantly from the acidified samples $(P<0.05)$.

The viscosities of the acidified xanthan and guar diets in solution fell significantly ( $P<0.05$ and $P<0.01$ respectively) with the addition of the neutralizing solution. Neutralizing the xanthan diet resulted in a lower viscosity than the similarly diluted saline control sample $(P<0.05)$, while there were no significant differences between the viscosities of the reneutralized and saline-diluted guar solutions. 


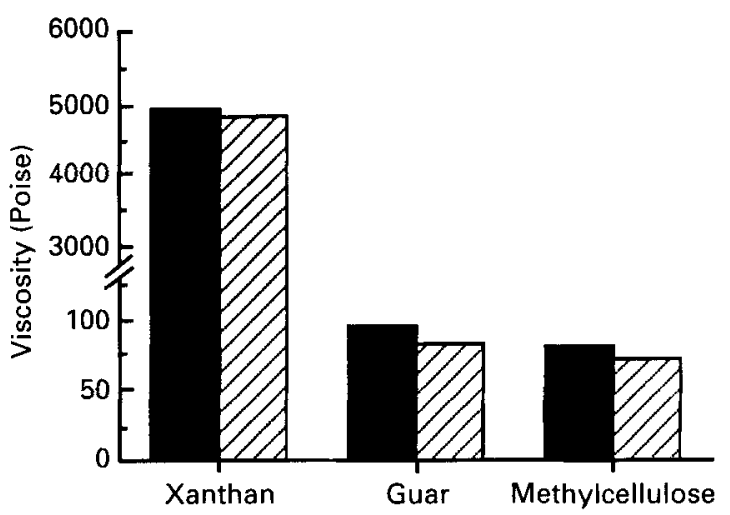

Fig. 1. Viscosity of the stomach ( $\square$ ) and small-intestinal $(\square)$ contents of rats given the experimental diets containing either xanthan gum, guar gum or methylcellulose. Digesta from the wheat bran-fed animals had no measurable viscosity. Results are single measurements on pooled samples from five rats. For details of diets, see Table 1.

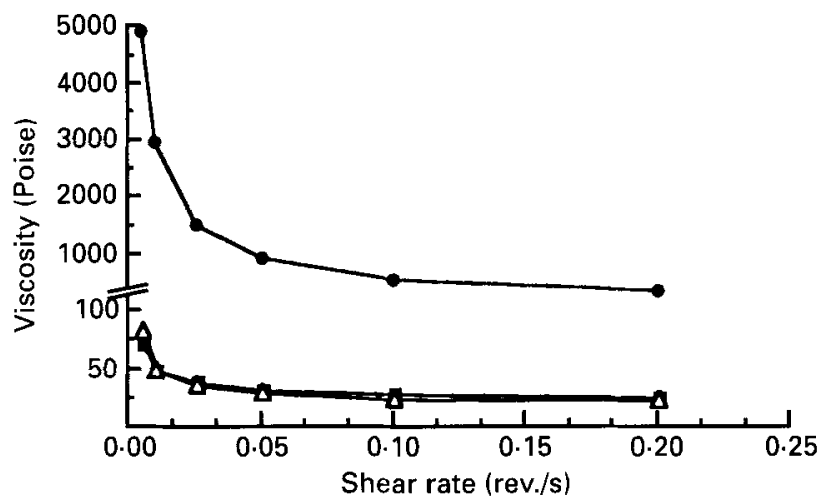

Fig. 2. Effect of shear on the viscosity of the small intestinal contents of rats given the experimental diets containing either xanthan gum $(\bullet)$, guar gum $(\triangle)$ or methylcellulose $(\boldsymbol{\square})$. Results are single measurements on pooled samples from five rats. For details of diets, see Table 1.

\section{Table 2. Viscosity (Poise) of experimental diets after acidification and reneutralization $\$$}

(Mean values with their standard errors for five determinations)

\begin{tabular}{|c|c|c|c|c|c|c|c|c|c|c|}
\hline \multirow[t]{2}{*}{ Concentration $(\%) \ldots$} & \multicolumn{2}{|c|}{$\begin{array}{c}\text { Initial } \\
1.8\end{array}$} & \multicolumn{2}{|c|}{$\begin{array}{c}\text { Acidified } \\
1.6\end{array}$} & \multicolumn{2}{|c|}{$\begin{array}{c}\text { Saline- } \\
\text { diluted } \\
\text { control } \\
1.6\end{array}$} & \multicolumn{2}{|c|}{$\begin{array}{c}\text { Reneutralized } \\
1 \cdot 24\end{array}$} & \multicolumn{2}{|c|}{$\begin{array}{c}\text { Saline- } \\
\text { saline- } \\
\text { diluted } \\
\text { control } \\
1.24\end{array}$} \\
\hline & Mean & SEM & Mean & SEM & Mean & SEM & Mean & SEM & Mean & SEM \\
\hline$x$ & 1210 & 34 & 2320 & 78 & 2218 & 34 & 1226 & $154 \dagger$ & 1970 & 84 \\
\hline G & 745 & 39 & 1008 & $52 \dagger$ & 691 & 39 & 263 & $12 \ddagger$ & 207 & 16 \\
\hline $\mathrm{MC}$ & 6.18 & 0.6 & $4 \cdot 41$ & $0 \cdot 4$ & $3 \cdot 81$ & $0 \cdot 3$ & $2 \cdot 87$ & $0.3 \pm$ & $2 \cdot 40$ & $0 \cdot 1$ \\
\hline
\end{tabular}

$\mathrm{X}$, Xantham gum; G, guar gum; MC, methylcellulose.

$\dagger$ Significantly different from controls; $P<0.05$.

$¥$ Significantly different from initial dilution; $P<0.01$.

$\S$ For details of diets and procedures, see Table 1 and pp. 564-565. 


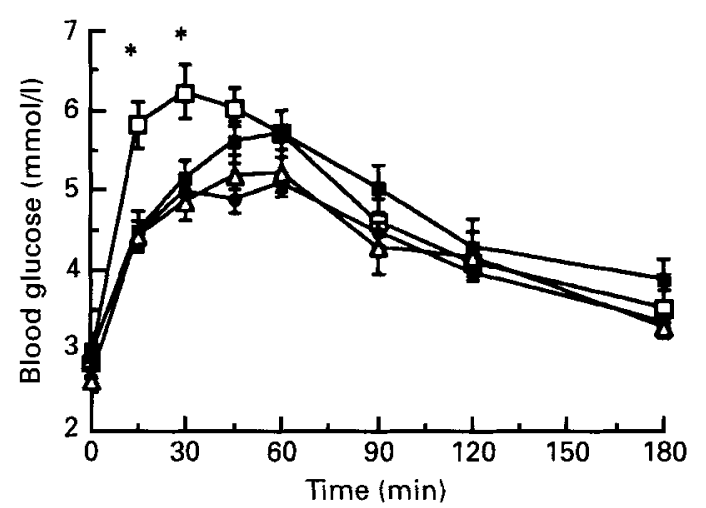

Fig. 3. Blood glucose response to an orally administered meal $(0.25 \mathrm{~g}$ carbohydrate $/ \mathrm{kg}$ body wt $)$ supplemented with $70 \mathrm{~g} / \mathrm{kg}$ wheat bran $(\square)$, guar gum $(\triangle)$, xanthan gum $(\odot)$ and methylcellulose $(\boldsymbol{G})$. Values are means with their standard errors for four rats. For details of diets, see Table $1 .{ }^{*}$ Significantly different from viscous soluble fibres; $P<0.05$.

The methylcellulose diet was significantly less viscous than the xanthan and guar diets at all dilutions. Each dilution of the methylcellulose diet significantly lowered the viscosity of the solution $(P<0.05)$, with no measurable effect of $\mathrm{pH}$ changes on the viscosity of the diet solution when compared with the saline-diluted control samples.

Dilution of the diets with the acidic and neutralizing solutions resulted in no change in the viscosity of the xanthan diet, while the same dilutions on the guar and methylcellulose diets lowered the viscosity by an average of $65 \%$ and $54 \%$ respectively from the initial viscosity of the diets (Table 2).

The viscosities of the in vitro diluted diets exhibited striking differences from the viscosity of the digesta. While xanthan was the most viscous fibre in vitro following dilution with both the acidic and neutral solutions, the difference in viscosity between the xanthan and guar diets was only approximately 5-fold, whereas xanthan was approximately 500 -fold more viscous in vivo. Similarly, despite very significant viscosity differences in the GI tract between guar and methylcellulose, when diluted in vitro such differences were not evident in the digesta. Furthermore, the viscosities of the digesta obtained from the stomach and small intestine for each of the fibre types when diluted $1: 1$ with water were of similar magnitude. This is unlike the large reductions in the viscosity that were measured for xanthan and guar diets when diluted with acidic and neutralizing solutions in vitro.

\section{Acute glycaemic response}

Fasting blood glucose concentrations before the meal were not significantly different between the experimental groups. Oral administration of $0.25 \mathrm{~g}$ carbohydrate from the experimental diets per $\mathrm{kg}$ body weight led to the significant elevation of blood glucose levels in all of the experimental groups, shown in Fig. 3. The non-viscous wheat-bran diet resulted in the largest postprandial glucose response with an incremental area under the glucose curve (0-90 min) of 338 ( $\mathrm{SE} 27$ ) $\mathrm{mmol} / 1$ per $\mathrm{min}$. The glucose response was significantly elevated relative to the viscous fibres at 15 and $30 \mathrm{~min}(P<0.01)$. Despite significantly differing viscosities measured in the stomach and small intestines of rats maintained on diets supplemented with one of the viscous fibres, the acute glycaemic response to a meal containing each of the fibres was similar. Xanthan resulted in the smallest incremental area under the glucose curve of 237 (SE 24) $\mathrm{mmol} / \mathrm{l}$ per min, however, this was not significantly greater than the glycaemic responses to guar and methylcellulose (290 (SE 38) and 316 (SE 14) $\mathrm{mmol} / 1$ per min respectively). 


\section{DISCUSSION}

This study clearly demonstrates the effectiveness of soluble fibre in increasing the viscosity of gastric and small intestinal contents when incorporated into the diet of rats. The increased viscosity of the digesta after soluble fibre supplementation in rats confirms previous reports (Blackburn \& Johnson, 1981; Topping et al. 1988; Ikegami et al. 1990). However, direct comparisons between studies of the viscosity of digesta cannot be made due to the differing fibre preparations used, concentrations in the diets, analysis and measurement techniques (Ellis et al. 1986). In the present study the viscosities of the small intestinal digesta were not predicted by viscosity measurements of the diet made before ingestion. Before ingestion the methylcellulose diet was very much less viscous (approximately 100-fold) than the guar diet which had in turn approximately half the viscosity of the xanthan diet. Following the consumption of the diets by the rats the measured viscosity in the diluted GI contents for the xanthan diet was approximately 500fold greater than that measured for the guar and methylcellulose diets. This clearly demonstrates the ability of the GI tract to modify the viscosity of the diets once ingested.

The ability of the GI tract to alter dramatically the viscosities of ingested soluble fibres may be mediated either by dilution from secretions entering the GI tract or through the $\mathrm{pH}$ changes elicited by these secretions. This was investigated in vitro by dilution of the diets with solutions approximating the $\mathrm{pH}$ of gastric and duodenal secretions. Dilution of the diets containing the viscous fibres with the acidic solution resulted in enhanced viscosity of the guar diet when compared with the similarly diluted saline control. When acidified, the viscosity of neither the xanthan nor the methylcellulose diet was altered from their similarly diluted saline controls.

Dilution of the diets with the neutralizing solution led to a significant reduction in the viscosity of the xanthan diet, when compared with the saline-diluted control. The viscosities of the guar and methylcellulose diets were similar to their saline-diluted controls. However, the capacity of changes in the $\mathrm{pH}$ to alter the viscosities of the diets was minor when compared with the effect of dilution on the resultant viscosity. Dilution of the xanthan diet from an initial concentration in the solution of $18 \mathrm{~g} / \mathrm{kg}$ to $12 \mathrm{~g} / \mathrm{kg}$ resulted in a small increase in viscosity whereas the same dilution of the guar and methylcellulose diets led to reductions in their viscosities of $65 \%$ and $54 \%$ respectively. These results would suggest that dilution of the diet by GI secretions is the major determinant of the changes in the viscosity of the diet following ingestion. These findings also question the value of viscosity measurements of diluted food as a means of predicting the viscosity and subsequent physiological action of a meal in the gut.

In the current study the lack of correlation between the viscosity of the diets diluted with the acidic and neutralizing solutions and the viscosity of the digestive contents may be largely due to the arbitrary dilutions of the diets made in the present study. No attempt was made to dilute the diets as they may be in the GI tract. Interestingly, it has been suggested that soluble fibre can influence the quantity of GI secretions diluting the digesta. In pigs fed on diets rich in either soluble fibre or insoluble fibre the soluble fibre diet let to an increase of $81 \%$ in pancreatic and $41 \%$ in bile secretion (Sambrook, 1981; Zebrowska et al. 1983). The action of differing soluble fibre types on the volume of GI secretions mixing with the digesta is unknown.

In the present study the postprandial glycaemic response to a meal containing the nonviscous fibre wheat bran and each of the soluble fibres was measured. When compared with the wheat-bran diet, all of the soluble fibres were able to lower the acute hyperglycaemia although the reductions in glycaemia were small and maintained for only $60 \mathrm{~min}$ postprandially. Additionally, in the present study the glycaemic response to each soluble 
fibre type was similar and failed to reflect the very large differences in the viscosity that were measured in the GI contents of the rats maintained on diets containing each of the fibres. This is consistent with a recent study in which a small dose of guar gum was as effective as larger doses in lowering the blood glucose response in healthy subjects (Torsdottir et al. 1989). Clearly, small alterations in the viscosity of the digesta will have little effect on the postprandial glycaemic response. Therefore, small alterations in the viscosity of a meal would also not be expected to have a significant impact on the resultant glycaemic response.

It is possible that measurement of the viscosity of the small intestinal and stomach contents may not reflect or predict the viscosity of the digesta at the mucosa-lumen interface. The soluble fibres investigated in the present study behave in a non-Newtonian manner in solution when subjected to shear stresses, with increasing rates of shearing reducing the apparent viscosity of the fibres in solution. Although little is known of the shear rates that exist in the small intestine, several studies suggest that the consumption of soluble fibre may increase the motility and, hence, the shear rates in the gastrointestinal tract. The motor activity of canine jejunum and duodenum after a viscous meal was associated with greater frequency of contractions which were propagated over a greater distance when compared with a non-viscous meal (Bueno et al. 1980; Schemann \& Ehrlein, 1986). In human subjects guar increased the jejunal motility pattern when compared with a lipid meal (Welch \& Wordling, 1986). Therefore the measured viscosity of the digesta may not reflect the actual viscosity in vivo.

In summary, soluble fibres which develop significant viscosity in vitro increase the viscosity of the stomach and small intestinal contents. The viscosity of the diet present in the small intestine differs from the measured viscosity before ingestion. The action of the GI tract to modify the viscosity of the diet is primarily due to the dilution of the diet by the GI secretions. Furthermore, the reductions in the postprandial glucose response to a meal containing one of each of the viscous fibre types did not reflect the viscosity of the diet measured in the digesta. The similarity of the glycaemic response suggests that the measured viscosity of the small intestinal contents was unable to predict the reduction in the glycaemic response to a meal. Therefore, the measurement of small changes in the viscosity of a meal may have little relevance to the effect of the meal on acute metabolic events in vivo.

The present study was supported by grants from Deakin University. D.C-S. is a recipient of an Australian Postgraduate Research Award. The authors acknowledge Miss Julie McRae for technical assistance, Dr Gary O'Loughlin and Miss Sue Thom (CSIRO Division of Wool Technology, Geelong) for use of the viscometer and Ms Anna Reynolds (Chemiplas, Australia) for the kind gift of the guar.

\section{REFERENCES}

American Institute of Nutrition (1977). Report of the American Institute of Nutrition ad hoc committee on standards for nutritional studies. Journal of Nutrition 107, 1340-1348.

Blackburn, N. A. \& Johnson, I. T. (1981). The effect of guar gum on the viscosity of the gastrointestinal contents and on glucose uptake from the perfused jejunum in the rat. British Journal of Nutrition 46, 239-246.

Blackburn, N. A.\& Johnson, I. T. (1983). The influence of guar gum on the movements of inulin, glucose and fluid in the rat intestine during perfusion in vivo. Pflügers Archiv 397, 144-148.

Blackburn, N. A., Redfurn, J. S., Jarjis, H., Holgate, A. M., Hanning, I., Scarpello, J. H. B., Johnson, I. T. \& Read, N. W. (1984). The mechanisms of action of guar gum in improving glucose tolerance in man. Clinical Science 66, 329-336.

Bueno, L., Praddaude, F., Fioramonti, J. \& Ruckebusch, Y. (1981). Effect of dietary fiber on gastrointestinal motility and jejunal transit in dogs. Gastroenterology 80, 701-707.

Caspary, W. F., Elsenhans, B., Sufke, U., Ptok, M., Blume, R., Lembcke, B. \& Creutzfeldt, W. (1980). Effect of dietary fiber on absorption and mortility. Frontiers in Hormone Research 7, 202-217. 
Ebihara, K., Masuhara, R., Kiriyama, S. \& Manabe, M. (1981). Correlation between viscosity and plasma glucose- and insulin-flattening activities of pectins from vegetables and fruits in rats. Nutrition Reports International 23, 985-992.

Edwards, C. A., Blackburn, N. A., Craigen, L., Davison, P., Tomlin, J., Sugden, K., Johnson, I. T. \& Read, N. W. (1987). Viscosity of food gums determined in-vitro related to their hypoglycemic actions. American Journal of Clinical Nutrition 46, 72-77.

Ellis, P. R., Morris, E. R. \& Low, A. G. (1986). Guar gum: the importance of reporting data on its physicochemical properties. Diabetic Medicine 3, 490-491.

Flourie, B., Vidon, N., Florent, C. H. \& Bernier, J. J. (1984). Effect of pectin on jejunal glucose absorption and unstirred layer thickness in normal men. Gut 25, 936-941.

Goulder, T. J., Alberti, K. G. M. M. \& Jenkins, D. A. (1978). Effect of added fiber on the glucose and metabolic response to a mixed meal in normal and diabetic subjects. Diabetes Care 1, 351-355.

Ikegami, S., Tsuchihashi, F., Harada, H., Tsuchihashi, N., Nishide, E. \& Innami, S. (1990). Effect of viscous indigestible polysaccharides on pancreatic-biliary secretion and digestive organs in rats. Journal of Nutrition $120,353-360$.

Jenkins, D. J. A., Leeds, A. R., Gassull, M. A., Cochet, B. \& Alberti, K. G. M. M. (1977). Decrease in postprandial insulin and glucose concentrations by guar and pectin. Annals of Internal Medicine 86, 20-23.

Jenkins, D. J. A., Wolever, T. M. S., Leeds, A. R., Gassull, M. A., Haisman, P., Dilawari, J., Goff, D. V., Metz, G. L. \& Alberti, K. G. M. M. (1978). Dietary fibres, fibre analogues, and glucose tolerance; importance of viscosity. British Medical Journal 1, 1392-1394.

Lund, E. K., Gee, J. M., Brown, J. C., Wood, P. J. \& Johnson, I. T. (1989). Effect of oat gum on the physical properties of the gastrointestinal contents and on the uptake of D-galactose and cholesterol by rat small intestine in vitro. British Journal of Nutrition 62, 91-101.

O'Connor, N., Tredger, J. \& Morgan, L. (1987). Viscosity differences between various guar gums. Diabetologia 20, 612-615.

Sambrook, I. E. (1981). Studies on the flow and composition of bile in growing pigs. Journal of the Science of Food and Agriculture 32, 781-791.

Schemann, M. \& Ehrlein, H. J. (1986). Postprandial patterns of canine jejunal motility and transit of luminal content. Gastroenterology 90, 991-1000.

Sherman, P. (1970). Industrial Rheology. London: Academic Press.

Topping, D. L., Oakenfull, D., Trimble, R. P. \& Illman, R. J. (1988). A viscous fibre (methylcellulose) lowers blood glucose and plasma triacylglycerols and increases liver glycogen independently of volatile fatty acid production in the rat. British Journal of Nutrition 59, 21-30.

Torsdottir, I., Alpsten, M., Andersson, H. \& Einarrsson, S. (1989). Dietary guar gum effects on postprandial blood glucose, insulin and hydroxyproline in humans. Journal of Nutrition 119, 1925-1931.

Welch, I. M“'. L. \& Worlding, J. (1986). The effect of ileal infusion of lipid on the motility pattern in humans after ingestion of a viscous, nonnutrient meal. Journal of Physiology 378, 12P.

Zebrowska, T., Low, A. G. \& Zebrowska, H. (1983). Studies on gastric digestion of protein and carbohydrate. gastric secretion and exocrine pancreatic secretion in the growing pig. British Journal of Nutrition 49, 401-410. 